\title{
Singular isothermal sphere and black hole formation
}

\author{
Mike J. Cai ${ }^{1}$ and Frank H. Shu ${ }^{2}$ \\ ${ }^{1}$ Institute of Astronomy and Astrophysics, Academia Sinica, Taiwan \\ email: mike@asiaa.sinica.edu.tw \\ ${ }^{2}$ Department of Physics, National Tsing-Hua University, Hsinchu, Taiwan
}

\begin{abstract}
We present here the self-similar collapse of a relativistic singular isothermal sphere (SIS) that leads to monolithic black hole formation. The dynamic evolution is triggered by the central portion of the SIS collapsing to for an infinitesimal black hole. This perturbation removes the pressure support for the layer immediately above, which causes it to collapse, and so on. The influence of gravitational collapse propagates out as an expansion wave moving at the speed of sound. Outside of the expansion wave, matter remains in local equilibrium.
\end{abstract}

\section{Introduction}

The discovery of high redshift QSO's at $z>6$ indicate that black holes of several billion solar masses already existed when the Universe was less than one billion years old (Fan et al. 2003). The standard picture of merger and accretion would take too long for a stellar mass black hole to grow to super-massive size. Thus, several scenarios of monolithic formation of black holes from primordial gas or relativistic star clusters have been proposed (see, e.g., Shapiro \& Teukolsky 1985). The formation process is divided into two phases. The first phase is the quasi-static evolution of the progenitor toward the singular configuration of gravothermal catastrophe. Numerical simulations suggest that the end state of gravothermal catastrophe can be described by singular isothermal equilibria (Balberg 2002). The second phase is the dynamic collapse triggered by gravitational or relativistic instability. In this work, we calculate the spacetime evolution of the second phase of dynamic collapse when the whole system is spherically symmetric.

\section{Basic Equations}

The most general spherically symmetric and self-similar spacetime can be written as

$$
d s^{2}=-\alpha^{2} d t 2+a^{2} d r 2+r^{2} d \theta^{2}+r^{2} \sin ^{2} \theta d \phi^{2},
$$

where $\alpha$ and $a$ are functions of the self-similar variable $\zeta=r / t$. For simplicity, we assume the matter content is a perfect fluid characterized by the isothermal equation of state $p=\gamma \rho$. The stress-energy tensor takes the usual form

$$
T_{\mu \nu}=\rho(1+\gamma) u_{\mu} u_{\nu}+\gamma \rho g_{\mu \nu} .
$$

Self-similarity allows us to define a non-dimensionalized density $\varepsilon(\zeta)=4 \pi r^{2} \rho$. A local Lorentz observer see the fluid having three-velocity $v=a u^{r} / \alpha u^{t}$. For computational purposes, we find it useful to work with

$$
\beta=-\frac{2 v}{1-v^{2}}, \quad x=\frac{a r}{\alpha t},
$$




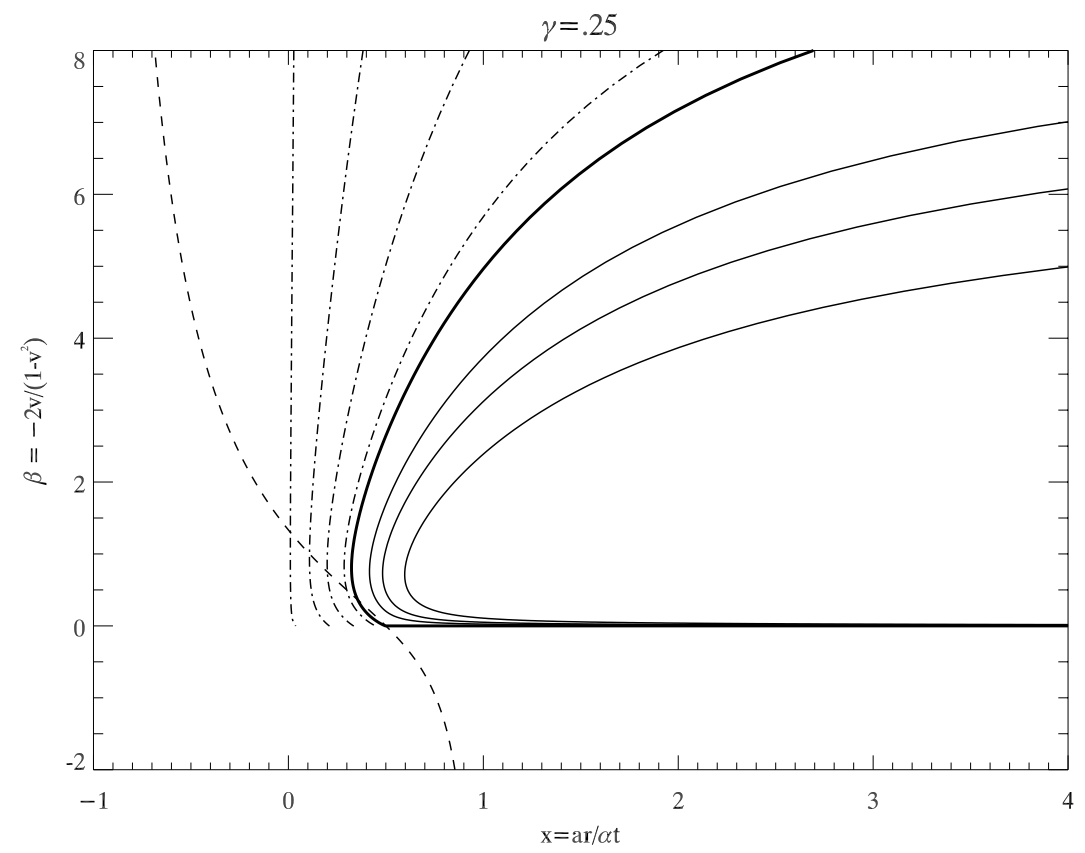

Figure 1. Self-similar solutions for a relativistic spherical collapse. The heavy solid curve represents the expansion-wave solution.

rather than $v, \alpha$, and $a$ directly. The variable $x$ has the obvious interpretation of proper self-similar variable. As an initial condition, we demand that velocity $v$ vanishes and the rescaled energy density $\varepsilon$ approaches a constant for sufficiently large value of $\zeta$ (i.e., when $r \rightarrow \infty$ or $\left.t \rightarrow 0^{+}\right)$.

\section{Results}

We plot here the velocity function $\beta$ versus the proper self-similar variable $x$. The dashed curve is the sonic surface. The solid light curves are systems where gravity overwhelms pressure gradient initially. Gravitational collapse occurs instantaneously at every radius. The dash-dotted curves are the solutions where pressure gradient is stronger than gravity. They represent time reversed self-gravitating wind. Between the two, we have our physical solution that corresponds to an inside-out collapse, represented by the heavy solid curve. The system was initially in an equilibrium configuration, which is the nonrotating limit of a singular isothermal toroid studied by Cai \& Shu(2003). At $t=0$, the central portion collapsed through its gravitational radius. Without pressure support, the layer immediately above starts to fall freely toward the event horizon, and the influence of gravitational collapse propagates out as an expansion wave. The expansion wave front is defined by $x=a r / a t=\gamma^{1 / 2}$, which is the isothermal sound speed.

\section{References}

Balberg, S., Shapiro, S. L., \& Inagaki, S. 2002, ApJ , 568, 475

Cai, M. J., \& Shu, F. H. 2003, ApJ, 583, 401

Fan, X., Strauss, M., Schneider, D., et al. 2003, AJ, 125, 1649

Shapiro, S. L., \& Teukolsky, S. A. 1985, ApJ, 292, L41 